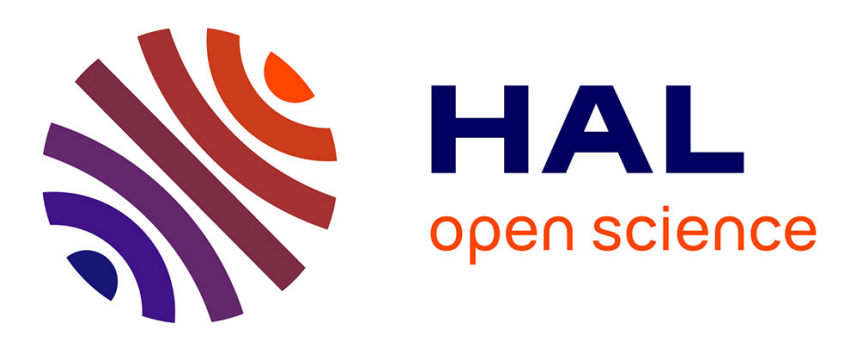

\title{
La fausse information au regard des vertus épistémiques de Wikipédia \\ Marie-Noëlle Doutreix
}

\section{To cite this version:}

Marie-Noëlle Doutreix. La fausse information au regard des vertus épistémiques de Wikipédia. Le Temps des médias. Revue d'histoire, 2018, La fausse information de la Gazette à Twitter, 30 (1), pp.91-104. 10.3917/tdm.030.0091 . hal-02169931

\section{HAL Id: hal-02169931 \\ https://hal.science/hal-02169931}

Submitted on 19 Dec 2020

HAL is a multi-disciplinary open access archive for the deposit and dissemination of scientific research documents, whether they are published or not. The documents may come from teaching and research institutions in France or abroad, or from public or private research centers.
L'archive ouverte pluridisciplinaire HAL, est destinée au dépôt et à la diffusion de documents scientifiques de niveau recherche, publiés ou non, émanant des établissements d'enseignement et de recherche français ou étrangers, des laboratoires publics ou privés. 


\title{
LA FAUSSE INFORMATION AU REGARD DES VERTUS ÉPISTÉMIQUES DE WIKIPÉDIA
}

\author{
Marie-Noëlle Doutreix
}

Nouveau Monde éditions | «Le Temps des médias »

2018/1 n 30 | pages 91 à 104

ISSN 1764-2507

ISBN 9782369426806

Article disponible en ligne à l'adresse :

https://www.cairn.info/revue-le-temps-des-medias-2018-1-page-91.htm

Distribution électronique Cairn.info pour Nouveau Monde éditions.

(C) Nouveau Monde éditions. Tous droits réservés pour tous pays.

La reproduction ou représentation de cet article, notamment par photocopie, n'est autorisée que dans les limites des conditions générales d'utilisation du site ou, le cas échéant, des conditions générales de la licence souscrite par votre établissement. Toute autre reproduction ou représentation, en tout ou partie, sous quelque forme et de quelque manière que ce soit, est interdite sauf accord préalable et écrit de l'éditeur, en dehors des cas prévus par la législation en vigueur en France. Il est précisé que son stockage dans une base de données est également interdit. 


\section{La fausse information au regard des vertus épistémiques de Wikipédia}

\section{Marie-Noëlle Doutreix ${ }^{\star}$}

Mettre en perspective l'idée de fausse information dans l'activité encyclopédique fait advenir une tension entre les notions d'information et de connaissance, en même temps qu'entre une prétention à la vérité et un risque de fausseté. Pourtant, il semble que Wikipédia tente de maintenir une certaine équivalence entre information et connaissance et mette à l'écart la valeur de vérité comme critère encyclopédique. L'épistémologie de Wikipédia substitue l'idée d'information vérifiable à celle d'information vraie, et la connaissance, assujettie de la même façon à la vérifiabilité comme principe de régulation ${ }^{1}$, ne semble pas s'en distinguer. Or "vérifiable» renvoie ici à ce qui a été énoncé par une source conforme, indiquée dans les notes et références de l'article. Comment la fausse information estelle pensée et impensée dans Wiki- pédia? L'épistémologie de la vérifiabilité informationnelle empêche-telle la formation d'informations fausses? À partir de l'analyse d'un article d'actualité et de sa page de discussion, dans laquelle les contributeurs débattent de l'éventuelle fausseté de certaines informations, nous dégagerons quelques procédés mis en œuvre par l'encyclopédie pour limiter la formulation et la pérennisation de fausses informations ${ }^{2}$. Nous chercherons les modes selon lesquels la fausse information est thématisée dans Wikipédia: est-elle stigmatisée comme erreur factuelle ou comme un manquement aux règles et principes liés à la vérifiabilité et à la neutralité ? Si tel était le cas, le glissement conceptuel éloignerait la question de la valeur de vérité des informations du problème de l'existence des faits qu'elles relatent.

\footnotetext{
^ Doctorante au sein du Centre d'Approches Pragmatiques en Philosophie du Langage et de la Communication (EA 1484 Communication Information Médias) et enseignante au département Institut de la Communication et des Médias de la Sorbonne Nouvelle.
} 


\section{L'information vérifiable}

Wikipédia se présente comme un projet encyclopédique ouvert à la participation de tous, tant en ce qui concerne la rédaction des articles que les modalités d'organisation de son activité éditoriale. À cette fin, le site de Wikipédia archive toutes les modifications des pages qu'il héberge, y compris les interactions entre contributeurs. Ces derniers assurent la gouvernance de Wikipédia et fixent ses règles dans la limite du respect des principes fondateurs de l'encyclopédie. Or la prétention à la vérité n'est pas portée par les principes fondateurs et l'idée de vérité est absente de la page consacrée au principe «Wikipédia est une encyclopédie $»^{3}$. Alors que les seules références à la notion de vérité dans les principes de l'encyclopédie résident dans l'énoncé du principe de neutralité, celles-ci servent à évacuer la question du vrai. En effet, aucun point de vue ne doit être présenté comme étant la vérité; de plus, la structure de l'article ne doit pas insinuer que le propos du passage principal est «vrai» et "non controversé", ni qu'un propos séparé dans une section ad hoc est "controversé", donc possiblement «faux $»^{4}$. Par conséquent, la vérité n'est pas la vertu épistémique ${ }^{5}$ prônée par Wikipédia et elle doit, selon les principes mêmes de l'ency- clopédie, s'effacer si elle n'est pas assez reconnue:

«Si un point de vue n'est partagé que par une minorité minuscule, il n'a pas sa place dans Wikipédia (sauf peut-être dans un article annexe), qu'il soit vrai ou non, et qu'un participant puisse le prouver ou non ${ }^{6} »$.

S'il est possible de lire dans la prudence vis-à-vis de l'idée de vérité un relativisme inspiré des travaux d'histoire et de philosophie des sciences ${ }^{7}$, la distanciation de la question du vrai et du faux est aussi un moyen circonstanciel pour que des personnes ayant des croyances différentes puissent écrire ensemble sur un même sujet ${ }^{8}$.

Dans l'article "Encyclopédie» de Wikipédia, reconnu «bon article» par la communauté wikipédienne, la notion de fausseté est absente de l'histoire de l'encyclopédisme et de sa conceptualisation, même en tant que limite. La vérité est quant à elle uniquement sollicitée à propos des ambitions de l'encyclopédisme: "Alors qu'elle aspire à dire le vrai sur toute chose, une encyclopédie n'est jamais à l'abri des biais culturels ou idéologiques de ses rédacteurs». D'après cette conception, l'aspiration à dire le vrai serait limitée par l'absence de neutralité. En effet, selon le principe de neutralité de Wikipédia, aspirer à dire le vrai implique de ne pas prendre 
parti: une encyclopédie restituerait parfois des informations fausses, mais rapporter les différentes conceptions en usage, même contradictoires, ferait partie de son honnêteté. Ainsi, à la question «Si nous étions quelques siècles en arrière, Wikipédia affirmerait-elle que le Soleil tourne autour de la Terre (tout en mentionnant rapidement les théories alternatives, en particulier celle d'un farfelu nommé Copernic) ?» posée en 2007 à la communauté wikipédienne $83,9 \%$ des votants ont répondu par l'affirmative'.

Aspirer à dire le vrai implique d'être sincère. En ce sens, l'attention n'est pas portée sur la valeur de vérité d'une proposition mais sur l'attitude épistémique du proposant ${ }^{10}$. Aussi, en tant que prétention, le vrai ne concernerait-il pas tant le contenu encyclopédique que les rédacteurs. Cette focalisation sur la sincérité ou plutôt sur la non-sincérité, qui est conçue par les wikipédiens comme du vandalisme, a un impact sur ce qui est considéré comme faux et sur l'acceptation de telle ou telle modification au sein d'un article.

La prétention au dire vrai est remplacée dans Wikipédia par la prétention au re-dire ${ }^{11}$. L'une des exigences fondamentales est de ne donner que des informations ou des idées qui ont déjà été publiées par un autre média. Cela permet de prendre la transparence des sources comme critère encyclopédique et de remplacer l'exigence de vérité par une exigence de vérifiabilité. Ici se dessine l'équivalence wikipédienne plus ou moins implicite entre connaissance et information, car ce qui est repris peut aussi bien consister en une série d'informations provenant d'articles de la presse généraliste qu'en des connaissances conçues et argumentées dans un cadre scientifique. La manière dont Wikipédia conçoit l'encyclopédie et le rapport de celle-ci à l'information est particulièrement visible dans le passage suivant:

«Plus que jamais, le public a besoin d'informations actualisées, appuyées sur des sources faisant autorité, facilement accessibles, et dont l'impartialité soit confirmée par l'arbitrage d'une diversité de contributeurs $[\ldots]^{12}$ ».

Tandis qu'une connaissance est censée correspondre à une proposition vraie, ce n'est pas le cas de l'information dont la valeur de vérité n'est pas prédéterminée au-delà du présupposé de véracité s'exerçant par défaut dans les interactions communicationnelles. La construction d'articles par «bouts d'informations ${ }^{13}$ " semble ainsi problématique dans la mesure où toute encyclopédie expose des connaissances et des savoirs, se distinguant par là même d'autres types de publications. 
Or, non seulement Wikipédia considère elle-même que son travail consiste à rassembler des informations, mais elle énonce comme principe que la question du vrai et du faux doit être écartée au profit de la vérifiabilité:

«Le critère qui détermine si une information peut être ajoutée dans Wikipédia n'est pas la question de savoir si elle est vraie ou fausse, mais de savoir si elle est attribuable à une publication vérifiable ${ }^{14} »$.

L'actualité est un sujet porteur de fausses informations dans Wikipédia mais cela est surtout significatif du fait que, pour certains types d'articles, Wikipédia est dépendante des sources journalistiques et que celles-ci diffusent parfois des informations inexactes. Une marge d'erreur pour les événements en cours et récents est reconnue par Wikipédia qui prévient le lecteur des éventuelles informations incorrectes par un bandeau apposé en début d'article. Si de fausses informations peuvent être présentes dans tous les sujets, que ce soit dans Wikipédia ou dans les médias journalistiques, il convient de noter que les débats sur la fausseté se déroulent dans les pages des articles dont les sujets provoquent des divergences idéologiques importantes, par exemple les sujets faisant l'objet de désaccords politiques dans l'espace public.
Information/contre-information, un modèle pour déjouer la fausse information?

L'article «Aéroport du Grand Ouest» (habituellement appelé "aéroport de Notre-Dame-des-Landes") revu 1778 fois par 392 rédacteurs depuis sa création le 3 avril 2008 a été considérablement travaillé de manière à respecter au mieux les principes de l'encyclopédie. Il présente depuis mai 2016 une certaine stabilité avec une moyenne de huit modifications par mois ${ }^{15}$. Néanmoins, le projet d'aéroport ayant pour l'instant un avenir incertain, à la fois dû à son statut de projet et aux controverses importantes dont il fait l'objet, un bandeau avertit le lecteur du caractère non avéré des informations exposées:

"Cet article contient des informations sur un projet en cours. Il se peut que ces informations soient de nature spéculative et que leur teneur change considérablement alors que les événements approchent».

La catégorie "Projet en cours», qui contient actuellement dix-sept articles, appartient elle-même à la catégorie "Événement en cours» qui en comporte plus de mille, chaque article présentant un bandeau d'avertissement. Sachant que de nombreux 
articles sont catégorisés «Événement en cours» avant d'être régularisés une fois l'événement fini, ce type de statut ne constitue pas un cas isolé dans Wikipédia. S'ajoutent de plus les catégories «Événement récent» et «Événement à venir» qui recoupent également les 1823 articles traitant de sujets d'actualité présentement rattachés au "Portail actualité».

La plupart des données concernant le projet d'aéroport divergeant selon les sources, l'article est construit sur le mode du discours rapporté. Cela lui permet entre autres d'avancer des chiffres sans prendre en charge leur valeur de vérité. L'autre méthode utilisée consiste à présenter pour chaque information controversée une contreinformation, comme nous désignerons toute information contredisant une information précédente et insérée afin de prendre place dans une pluralité de points de vue. Ladite contre-information peut se situer juste après l'information controversée ou dans une autre section réservée aux contreinformations. Dans l'article, la parole de ceux qui se positionnent comme parties prenantes du projet d'aéroport joue le rôle d'information et celle de ceux ayant un point de vue négatif sur la construction d'un aéroport à Notre-Dame-des-Landes passe pour une forme de contre-information. Dans quelle mesure ces deux procédés, celui du discours rapporté et celui du couple information/contre-information, participent-ils du modèle de la vérifiabilité associée à la neutralité de point de vue?

\section{La structuration des points de vue et leur attribution respective}

L'introduction de l'article «Aéroport du Grand Ouest» illustre la méthode wikipédienne pour évacuer la question du vrai et du faux: composée de quinze phrases, elle présente quinze références, c'est-à-dire l'équivalent d'une référence par phrase bien que trois phrases ne mentionnent aucune référence et que deux en contiennent plusieurs. Cette abondance de sources est à l'image de l'ensemble de l'article qui ne comprend pas moins de deux cent quatre-vingt-treize notes et références. Une telle façon d'observer un respect scrupuleux de la règle de vérifiabilité parvient-elle à écarter les problèmes de vérité à la fois dans le contenu de l'article et dans la perception qu'en ont les contributeurs?

Parmi les quinze références, sept références officielles promeuvent le projet d'aéroport (sites de la ville de Nantes, de la Préfecture de LoireAtlantique, du syndicat mixte aéroportuaire et quatre références du site du projet d'aéroport) et huit sources proviennent de médias reconnus. Au sein de ces sources journalistiques, 
seul Politis est engagé sur les questions environnementales. Les sources mobilisées dans l'introduction apparaissent donc majoritairement en accord avec le projet d'aéroport. Y figure néanmoins un point litigieux portant sur le coût du nouvel aéroport. En effet, dans le paragraphe consacré à cette question au sein de l'introduction, seul est indiqué le coût estimé par les porteurs du projet datant de 2010, alors même que depuis 2013 ce coût a été revu à la hausse par ces mêmes sources. Les «opposants» sont cités pour notifier que ce coût officiel est contesté mais leur point de vue, selon lequel d'autres coûts que ceux reconnus dans le projet devraient être pris en compte, est lui-même nuancé ${ }^{16}$. De plus, sans données précises, ce désaccord parait anecdotique alors que, plus loin dans l'article, est écrit qu'en intégrant ces coûts l'estimation du projet passerait de 556 millions d'euros à 4 milliards d'euros. Ces questions de chiffres, qui ont l'apparence du factuel, constituent un grand enjeu en termes d'argumentation et ainsi un terrain propice aux fausses informations car les coûts allégués du nouvel aéroport sont mobilisés en faveur ou en défaveur du projet.

La question financière est débattue dans la page de discussion attachée à l'article "Aéroport du Grand Ouest». Un contributeur intervient pour dés- approuver le fait de laisser uniquement dans l'introduction l'estimation du coût de l'aéroport donnée par ses promoteurs alors qu'une autre évaluation serait reléguée vers une section «débat» car cela suggérerait que l'estimation des promoteurs est la seule donnée objective. En effet, seul le coût officiel (le plus bas de ceux figurant dans l'article) est indiqué dans l'introduction alors que des chiffres différents sont évoqués dans les autres parties ${ }^{17}$. Son intervention répond à celle d'un autre contributeur pour qui l'article manque de neutralité. Ce dernier souhaite que les "polémiques" sur le coût de l'aéroport soient déplacées dans la section présentant les différents débats. Or considérer que les désaccords sur le coût sont des polémiques qui doivent être présentées à part revient déjà à juger que l'estimation des "opposants» n'est pas une information en soi mais un point de vue. Celle-ci a pourtant une référence, Médiapart, mais le contributeur critique cette référence car elle n'est lisible que sous abonnement. Néanmoins, si cette restriction rend l'information moins facilement vérifiable, elle ne l'invalide pas.

Dans la section «Rénovation de l'aéroport actuel de Nantes Atlantique» de la partie "Autre solution au projet", il est écrit «À l'automne 2013, la DGAC estime que le coût d'une 
rénovation de l'aéroport actuel serait assez proche de celui du transfert». Sachant que les coûts de transfert sont évalués de 556 millions d'euros à 4 milliards d'euros selon les sources mentionnées dans l'article, l'information semble équivoque. L'article indiqué pour justifier l'idée d'une équivalence des coûts, provenant de la version en ligne du quotidien régional de Bretagne Le Télégramme et intitulé «N.-D. des Landes. La DGAC chiffre les projets", affirme que l'aménagement de l'aéroport actuel pour augmenter son trafic coûterait au total 825 millions d'euros et que :

«L'aéroport de Notre-Dame-desLandes, s'il était calibré pour 9 millions de passagers, reviendrait, lui, à 807 millions d'euros rien que pour le génie civil et la navigation aérienne, sans compter les achats de terres depuis le début du projet, qui n'ont pas été chiffrés ${ }^{18} »$.

Reprenant les paroles du directeur du transport aérien à la DGAC, l'article en conclut que l'adaptation de l'ancien aéroport coûte presque aussi cher que la construction du nouveau.

Au-delà du fait que l'article de presse référencé annonce des chiffres qui ne correspondent à aucun de ceux évoqués dans l'article de Wiki- pédia, il annonce des informations opposées à celles publiées la veille par le même journal. En effet, un article s'appuyant pourtant sur la même étude de la DGAC titrait «Notre-Dame-des-Landes. L'adaptation de l'actuel aéroport coûterait plus cher ${ }^{19}$ !» ce qui s'oppose à l'article référencé dans Wikipédia disant qu'adapter l'aéroport coûte un peu moins cher que d'en construire un nouveau (même en prenant une estimation basse du coût de "transfert»). L'article intitulé «Notre-Dame-desLandes. L'adaptation de l'actuel aéroport coûterait plus cher!» annonçait ainsi dès son titre une information fausse, elle-même justifiée par une information sur le coût du nouvel aéroport datant de 2010 et reconnue comme inexacte en l'état actuel:

«Le maintien, en l'adaptant, de l'actuel aéroport de Nantes coûterait plus cher que son transfert vers le site contesté de Notre-Dame-desLandes, affirme la Direction Générale de l'Aviation Civile (DGAC), dans une étude réalisée à la demande de la commission du dialogue et rendue publique ce mercredi par la préfecture de Loire-Atlantique. Dans une étude rendue publique ce mercredi, la DGAC indique que pour adapter l'actuel aéroport de Nantes Atlantique, les travaux envisagés coûteraient 685 millions d'euros. Il faudrait y 
ajouter 140 millions de travaux préparatoires et de pertes d'exploitation, soit un total de 825 millions d'euros. À comparer au coût de 556 millions d'euros de l'aéroport de Notre-Damedes-Landes, tel qu'estimé en 2010, au moment de l'attribution de sa concession au groupe Vinci, avant les aménagements du projet demandés en avril 2013 par la commission du dialogue ${ }^{20} . »$

Or dans le rapport de la DGAC il n'est écrit à aucun moment que les coûts de la construction d'un nouvel aéroport seraient de 556 millions ni que «le maintien, en l'adaptant, de l'actuel aéroport de Nantes coûterait plus cher que son transfert ${ }^{21}{ }^{\prime}$. Le Télégramme n'a pas démenti officiellement que son article du 6 novembre 2013 titrait une information fausse et l'article est toujours disponible en ligne sans qu'il y ait eu une rectification.

Notons de plus que l'article de Wikipédia souligne que le CéDpa est opposé au projet mais n'indique pas que la DGAC soutient le projet. De même, alors que la signification de CéDpa (Collectif des élus Doutant de la pertinence du projet d'aéroport) est spécifiée à trois reprises, il n'est pas indiqué que DGAC signifie Direction Générale de l'Aviation Civile, alors que l'acronyme est explicité dans l'article source. Cela a pour conséquence que telle information passe pour orientée, voire suspecte, parce que la position anti-aéroport est soulignée tandis que la position pro-aéroport, n'étant pas forcément précisée, passe pour objective.

\section{Le problème de la valeur des sources}

Le Télégramme a publié sur son site Internet de nombreux articles sur Notre-Dame-des-Landes, au moins vingt-neuf pour le mois de novembre 2016. Wikipédia a repris l'article du 7 novembre et non celui du 6 novembre qui comportait la fausse information la plus visible mais cinq articles du Télégramme sont tout de même cités dont la plupart ne sont que des brèves non signées. L'un d'entre eux, "Notre-Dame-des-Landes. Les chasseurs donnent de la voix ${ }^{22}$ " entièrement repris à un article du journal Presse Océan ${ }^{23}$, est constitué seulement du paragraphe suivant:

«Dans un courrier adressé le 14 janvier au préfet de Loire-Atlantique et que cite Presse Océan, la société des chasseurs deVigneux-de-Bretagne exprime son "ras-le-bol général", assurant que "propriétaires, actionnaires, sociétaires font l'objet d'agressions verbales et maintenant physiques", avec dépôt de plainte à la clé. La lettre incrimine les anti-aéroport établis sur place. Ils "se permettent de couper des 
arbres sur des propriétés privées sans autorisation", "dégradent les pelouses, font leurs besoins dans les miradors de chasse et les arrachent", "crèvent les pneus des remorques dédiées au ramassage du bois, écrit la société de chasse. Ils n'hésitent pas à couper les clôtures pour passer au travers de propriétés privées, laissent leurs véhicules et vélos dans les chemins".»

Cet article du Télégramme a ainsi comme seule source un autre article qui invoque lui-même comme source un courrier envoyé par une société de chasseurs, repris sans aucune distance tout en soulignant que la lettre incrimine «les anti-aéroport». Or Wikipédia reprend quasiment mot pour mot cette information mais enlève les guillemets présents dans les deux articles de presse et qualifie à cette occasion les anti-aéroport "d'éléments incontrôlables», expression qui ne figure dans aucun des deux articles cités.

Ce passage a été mis en cause dans la page de discussion par un contributeur désignant ces sources primaires «à la limite de la crédibilité» et qui compare plus loin l'article à une revue de presse. Un autre Wikipédien approuve cette prise de position et supprime le passage qui, pour lui, "n'a pas sa place sur Wikipédia». Il met de plus en exergue l'asymétrie dans le traitement des informations au sein de l'article, qu'exemplifie l'exigence d'apporter quelque chose de concret pour les informations provenant des anti-aéroport, exigence qu'il considère n'être pas respectée dans le passage relatant les plaintes des chasseurs. Un autre contributeur réplique en arguant de la pertinence du paragraphe, de la valeur du témoignage des «riverains mécontents» et accuse son interlocuteur d'attribuer une valeur plus grande aux informations des uns qu'aux informations des autres. Selon lui, ceux qui critiquent les méthodes journalistiques citent des journaux lorsque ceux-ci vont dans leur sens. Le fait qu'il déclare qu'"on n'est pas ici pour juger du bien-fondé de ces plaintes " est symptomatique d'un refus d'évaluer ou de questionner la qualité des articles journalistiques et la véracité des informations qu'ils relaient.

Cette attitude s'inscrit dans la continuité des principes de fonctionnement de l'encyclopédie qui mettent à distance la question du vrai et du faux. Le discours rapporté semble être le lieu idéal de la fausse information en ce qu'il est protégé du soupçon de partialité par la marque explicite d'emprunt à une source journalistique, même quand celle-ci ne présente elle-même aucun des signes habituels d'un travail sérieux (référence, recou- 
pement des sources, signature de l'article, effort d'analyse). Mais si le bienfondé d'une information de Wikipédia tient, pour les contributeurs, à sa présence dans un média journalistique, le bien-fondé des médias journalistiques repose parfois in fine sur des sources primaires comme des témoignages, où la question de la véracité ne peut plus être remplacée par la «vérifiabilité» d'une source secondaire. Or cette ambivalence n'est pas prise en compte par le contributeur qui clôt le débat en réaffirmant l'autorité des journaux qu'il cite ${ }^{24}$. Le passage litigieux est rétabli et figure encore dans l'article où aucune contre-information ou nuance n'est avancée à ce propos. En effet, le paragraphe d'après, qui conclut la section «Occupation de la Zad", surenchérit à propos d'une dégradation de matériels à l'encontre de chercheurs mandatés par le promoteur du projet d'aéroport, à l'aide d'un autre article du quotidien nantais Presse Océan et d'un article de 20 minutes. Ces accusations échappent ainsi à la pondération des différents points de vue, garantie de neutralité dans le système wikipédien.

\section{Une équivalence entre fausseté et partialité}

Si la mise à distance de la question du vrai et du faux autorise à relater des informations fausses sur le mode du discours rapporté, elle permet aussi en théorie de limiter certains cas problématiques comme ceux, prohibés par l'encyclopédie, où un contributeur fait reposer une information sur son propre témoignage. Ainsi, un contributeur non enregistré et dont les seules contributions semblent porter sur l'article «Aéroport du Grand Ouest» écrit dans la page de discussion:

«De toute façon beaucoup de militants sont sur Wikipédia pour faire de la désinformation. Impossible d'enlever des parties fausses, sans sources ou avec des sources non fiables anciennes, dont un raisonnement logique permettrait de déduire que c'est faux. [...] Quand on voit quelqu'un militant à gauche (et ouvertement sur son profil), rajoutant des choses contre l'aéroport sans source fiable... (Alors qu'on sait que c'est faux quand on y travaille).»

Ses accusations de fausseté, qu'il justifie par sa situation professionnelle, semblent concerner l'information selon laquelle la compagnie Airbus souhaiterait conserver la piste de Nantes-Atlantique pour transférer des pièces entre ses différentes usines. Le même argument, où le manque de sources est prétexté comme problème et où l'expérience personnelle prétend 
être porteuse de vérité, revient dans la page d'historique de l'article pour légitimer la suppression du passage dénoncé (suppression elle-même révoquée et réitérée plusieurs fois).

Le discours du contributeur exemplifie l'équivalence posée entre fausseté et partialité. Si un militant ajoute une information sans "source fiable", celle-ci est d'emblée suspecte ${ }^{25}$. Or la valeur de vérité de l'information concernant Airbus est particulièrement importante. Si des avions continuent d'utiliser l'actuel aéroport malgré la construction du nouvel aéroport, alors dire qu'il s'agit d'un transfert de l'aéroport et non de la construction d'une nouvelle structure devient faux puisque les deux aéroports seraient ainsi en activité. Cela rendrait également ambivalentes d'autres propositions énoncées dans l'article «Aéroport du Grand Ouest» :

«Fin 2007, la construction de l'aéroport du Grand-Ouest est confirmée, en dépit du Grenelle de l'environnement annonçant le gel de toute nouvelle structure aéroportuaire. Le nouvel aéroport a été jugé par la préfecture de Loire-Atlantique compatible avec les objectifs du développement durable s'agissant d'un transfert pour raisons environnementales et non de la création d'une infrastructure supplémentaire.»
Le terme «transfert» est celui utilisé par les pouvoirs exécutifs ${ }^{26}$ et qu'il s'agisse ou non réellement d'un transfert, le terme est adéquat selon les principes mêmes de Wikipédia, puisqu'employé par les sources faisant autorité sur le sujet, les sources officielles parties prenantes du projet d'aéroport. C'est dans cette même logique que l'article "Aéroport du Grand Ouest» n'est pas intitulé «Projet d'aéroport de Notre-Dame-desLandes» alors même que la règle de moindre surprise, en vigueur sur Wikipédia, aurait favorisé cette appellation. Dans la page de discussion, un contributeur de l'article remet néanmoins en cause l'idée que les sources officielles valent comme vérité indiscutable.

Dans les articles de Wikipédia concernant l'actualité, constater une proposition fausse revient souvent à accuser de partialité. Ainsi, la fausse information est ici porteuse d'une signification particulière car elle se double d'un jugement sur l'attitude épistémique de son auteur. Lors des cas litigieux, Wikipédia se limite à recycler des informations journalistiques:

"Cette situation est caractéristique du traitement des sujets controversés au sein du dispositifWikipédia débouchant soit sur une situation d'impasse où les termes des échanges se dété- 
riorent, soit sur des contenus réduits à des faits auparavant rendus publics par un travail de médiation journalistique ${ }^{27} »$.

En cas de conflit d'édition, la médiation ne vise pas à établir une vérité mais à rééquilibrer les points de vue.

Qu'est-ce que les principes et pratiques mettant en jeu la fausse information disent de Wikipédia en tant qu'encyclopédie? Ce n'est pas tant la présence de fausses informations qui pose question mais plutôt le contournement de la fausseté comme sujet de réflexion et d'organisation au profit d'un total respect des discours provenant des diverses sources agréées. Dans les articles traitant de sujets d'actualité dissensuels, la fausse information est une nouvelle, attestée ou non, perçue comme orientée idéologiquement par ceux qui la jugent fausse. Elle témoigne souvent de deux visions incompatibles. Si dans les médias la fausse information involontaire peut être liée à un manque de vérification journalistique, elle peut être due sur Wikipédia à la reprise des discours médiatiques et plus spécifiquement au fait que la vérification repose seulement sur le fait que tel ou tel propos a bien été énoncé par un média connu. Repoussant ainsi la vérité à un niveau où elle n'est pas elle-même prise en charge. En effet, comme Wikipédia prétend ne pas avoir à étayer la vérité d'une proposition ou l'existence d'un fait, cette démonstration est mise à la charge des sources citées. Néanmoins, ces sources peuvent elles-mêmes se décharger de la question de la vérité en la laissant aux témoins. Ce phénomène, visible dans le cas de la reprise d'articles de presse fondés sur un courrier écrit par une société de chasseurs, est symptomatique du traitement de l'information.

La fausse information constitue pour les rédacteurs des articles d'actualité ce qui manque au respect des règles de vérifiabilité et de neutralité, ces deux vertus épistémiques définissant le traitement de l'information dans Wikipédia. Néanmoins, la question de la fausse information pourrait se poser différemment dans les articles scientifiques très spécialisés car ceux-ci mobilisent seulement quelques contributeurs et leurs pages de discussion sont peu utilisées. Les vertus épistémiques des disciplines concernées s'y substitueraient-elles, dans les discours ou dans les pratiques, aux vertus soutenues par les principes fondateurs de Wikipédia? Cela conduirait à interroger l'éventuelle pluralité des épistémologies convoquées dans ce projet encyclopédique. 


\section{Notes}

1 «Un article présente les connaissances établies sur un sujet précis, connaissances qui doivent donc être vérifiables", voir la page «Wikipédia:Wikipédia est une encyclopédie».

${ }^{2}$ Plusieurs études se sont intéressées à la part de fausses informations dans Wikipédia, comme celle publiée dans Nature qui compare les erreurs dans Wikipédia et dans l'Encyclopaedia Britannica: Jim Giles, «Internet encyclopaedias go head to head", Nature, volume 438, 7070, 2005, p. 900-901. Ces études visaient davantage à évaluer Wikipédia et à questionner ou défendre sa crédibilité qu'à analyser les ressorts de la fausse information.

${ }^{3}$ Voir la page «Wikipédia: Wikipédia est une encyclopédie».

${ }^{4}$ Voir la page «Wikipédia: Neutralité de point de vue».

${ }^{5}$ Les vertus épistémiques sont des normes internalisées appliquées au nom de valeurs éthiques mais aussi au nom d'une efficacité pragmatique garantissant un savoir, Lorraine Daston, Peter Galison, Objectivité (2007), Dijon, Les presses du réel, 2012, p. 53.

${ }^{6}$ Voir la page «Wikipédia: Neutralité de point de vue».

${ }^{7}$ « Le but n'est donc pas de trouver une vérité ou une norme qui de toute façon évoluent selon l'endroit, le groupe et le temps. ", Ibid.

${ }^{8}$ La règle de vérifiabilité et le principe de neutralité évacuent la question du vrai et du faux pour favoriser un accord entre les contributeurs. Marie-Noëlle Doutreix, "Quel dialogisme dans l'écriture encyclopédique de Wikipédia?» in Marie-Dominique Popelard (dir.), La reprise en actes,
Rennes, Presses Universitaires de Rennes, 2017.

${ }^{9}$ Voir la page «Wikipédia: Sondage/Wikipédia et la Vérité».

${ }^{10}$ Cette interprétation est confirmée par la suite de la phrase "alors qu'elle aspire à dire le vrai sur toute chose, une encyclopédie n'est jamais à l'abri des biais culturels ou idéologiques de ses rédacteurs", voir l'article «Encyclopédie» de Wikipédia.

${ }^{11}$ Sur la notion de reprise, notamment discursive, voir Marie-Dominique Popelard (dir.), La reprise en actes, op. cit.

${ }^{12}$ Voir l'article «Encyclopédie» de Wikipédia.

${ }^{13}$ La connaissance est envisagée dans Wikipédia comme un ensemble de «bouts d'informations mis en sens par un travail collectif», Pierre Willaime, «Une analyse épistémologique de l'expertise dans Wikipédia", in Lionel Barbe, Louise Merzeau, Valérie Schafer (dir.), Wikipédia, objet scientifique non identifié, Paris, Presses Universitaires de Paris X, 2015, p. 105.

${ }^{14}$ Voir la page "Wikipédia: Citez vos sources".

15 Moyenne calculée pour la période de mai à décembre 2016.Voir la page "Auteurs et statistiques» correspondant à l'article.

16 «D'après les opposants, l'estimation avancée serait sous-estimée et le coût des constructions ferroviaires accompagnant le projet d'aéroport (qui ne seront toutefois pas destinées exclusivement à l'aéroport) devrait être pris en compte». Voir l'article «Aéroport du Grand Ouest». L'article a été rédigé fin 2017 avant que ne soient connues les décisions du gouvernement français le 17 janvier 2018.

${ }^{17}$ Dans la section «Financement de l'aéro- 
port» l'estimation du coût, attribuée au syndicat mixte aéroportuaire du Grand Ouest, est de 561 millions d'euros. D'autres chiffres sont avancés dans l'article: «Le coût total pourrait approcher les 3 milliards d'euros, selon le CéDpa, opposé au projet».

${ }^{18}$ Article non signé, «N.-D. des Landes. La DGAC chiffre les projets", Le Télégramme, 7 novembre 2013.

${ }_{19}$ Article non signé, «Notre-Dame-desLandes. L'adaptation de l'actuel aéroport coûterait plus cher!», Le Télégramme, 6 novembre 2013.

${ }^{20}$ Ibid.

${ }^{21}$ Direction générale de l'aviation civile, Évaluation du réaménagement de Nantes Atlantique dans le scénario d'un maintien de l'activité, Rapport d'étude, novembre 2013, http: / / www.develop pementdurable.gouv.fr/IMG/pdf/V2-12_NANTAT-RAPETU-131114.pdf.

${ }^{22}$ Article non signé, "Notre-Dame-desLandes. Les chasseurs donnent de la voix", Le Télégramme, 5 février 2013.

${ }^{23}$ Article non signé, «N-D.-des-Landes.
Cohabitation parfois rude entre antiaéroport et riverains", Presse Océan, 4 février 2013.

${ }^{24}$ «Les sources que j’ai citées sont celles de journaux réputés pour leur professionnalisme, leur indépendance d'esprit et leur objectivité (Ouest-France, Presse Océan et Le Télégramme) lus par des centaines de milliers de lecteurs dans le nord-ouest de la France. Donc $[\ldots]$ je ne vois pas où est le problème".

${ }^{25}$ Comme l'atteste aussi cet autre exemple provenant de la page de discussion «De plus, le chiffre de 150 élus n'est pas sourcé donc tu te bats pour la sauvegarde d'une contribution qui te semble admissible mais tu n'as pas l'air de te soucier de sa vérifiabilité (elle est vraie au moins cette affirmation? On n'a toujours pas la réponse)».

${ }^{26}$ Alain Gérardot-Paveglio, Transfert de l'aéroport de Nantes Atlantique sur le site de Notre-Dame des Landes, Service de la communication interministérielle, Préfecture de la Loire-Atlantique, 16 juillet 2009.

${ }^{27}$ Julien Levrel, «Wikipedia, un dispositif mediatique de publics participants", Reseaux, 138, 2006/4, p. 215. 